stem cells have appropriated this type of physical protection in fish.

It remains to be seen whether HSPCs home to locations protected by melanocytes, or whether melanocytes can be recruited or stimulated to proliferate by signals from blood stem-cell niches. Another question is why HSPCs seek out a different niche in terrestrial vertebrates. HSPCs regularly leave their niches and circulate in the blood, whereas other adult stem cells, although capable of movement, tend to be more static. Indeed, Kapp et al. highlighted this mobility in adult Rana frogs, in which the location of HSPCs switches depending on the season - perhaps driven by changing exposure to UV. This mobility could give HSPCs an increased capacity to seek out alternative sites that can better protect them from damage. Bone would completely encapsulate the stem cells, providing a shield from all angles. There might also be other benefits to a niche in the bone marrow, such as lower oxygen levels, which could provide protection from other forms of DNA damage.

Fish have evolved the ability to mitigate UV-induced DNA damage through lightdependent DNA repair ${ }^{10}$. The fact that, despite this ability, HSPCs seem to be under evolutionary pressure to seek additional protection highlights the importance of maintaining the fidelity of tissue stem cells. Physical protection might be of particular value in the haematopoietic system, because blood cells have a high turnover rate. If HSPCs are unable to re-establish bloodcell populations, the organism would be likely to die from anaemia or infection.

Finally, it might be predicted that other tissue-specific stem cells that have less migratory potential than HSPCs would be more prone to damage, because they would be less able to move to protective niches. By gaining insight into the evolutionary steps taken to protect haematopoietic stem cells, we might be able to develop strategies to protect these cell types, refining their current niches to better maintain stem-cell potential in longer-lived organisms, including humans. -

Isabel Beerman is at the National Institute on Aging, Biomedical Research Center, Baltimore, Maryland 21224, USA.

e-mail:isabel.beerman@nih.gov

1. Kapp, F. G. et al. Nature 558, 445-448 (2018)

2. Ferraro, F., Celso, C. L. \& Scadden, D. Adv. Exp. Med. Biol. 695, 155-168 (2010).

3. Orkin, S. H. \& Zon, L. I. Cell 132, 631-644 (2008)

4. Williams, M. J. J. Immunol. 178, 4711-4716 (2007)

5. Evans, C. et al. Adv. Dev. Biol. 18, 259-299 (2007).

6. de Jong, J. L. \& Zon, L. I. Annu. Rev. Genet. 39, 481-501 (2005).

7. Murayama, E. et al. Immunity 25, 963-975 (2006).

8. Martinez-Agosto, J. A., Mikkola, H. K., Hartenstein, V. \& Banerjee, U. Genes Dev. 21, 3044-3060 (2007).

9. Horton, J. D. (ed.) Proc. Symp. Development and Differentiation of Vertebrate Lymphocytes 1979 (Dev. Immunol. Vol. 8; Elsevier, 1980)

10.Sinha, R. P. \& Häder, D. P. Photochem. Photobiol. Sci. 1, 225-236 (2002).

This article was published online on 13 June 2018.

ASTRONOMY

\title{
Missing matter found in the cosmic web
}

The location of nearly half of the ordinary matter in the Universe is unknown. $X$-ray observations suggest that this elusive 'baryonic' matter is hidden in the filamentary structure of the cosmic web. SEE LETTER P.406

\section{TAOTAO FANG}

W e live in a dark Universe: just 5\% of it consists of ordinary matter such as that found in atoms, whereas the rest is 'dark' matter and energy that cannot currently be detected directly ${ }^{1}$. However, observations of the nearby Universe suggest that up to $40 \%$ of this ordinary matter - which is made up primarily of particles known as baryons - is missing ${ }^{2-5}$. Baryonic matter is thought to be distributed through the Universe like a cosmic web, and the missing baryons are predicted to be located in the filamentary structures that connect the web, and in intergalactic space ${ }^{4}$. On page 406 , Nicastro et al. ${ }^{6}$ report the detection of the $\mathrm{X}$-ray absorption signatures of baryons in the spectra of a bright background object. The findings might finally reveal a major reservoir for baryonic matter.

Why have the missing baryons been so difficult to detect? One reason is that the density of the baryonic matter in the filaments is extremely low. The other reason is that the high temperature in the filaments causes the most abundant element (hydrogen) to be almost completely ionized - which means that it has no electrons to produce spectral features that could be used to detect it. However, there might be trace amounts of heavier elements such as oxygen, in which a few electrons are bound. These ions can produce detectable (but extremely weak) spectral features, typically in the X-ray and/or ultraviolet regions of the electromagnetic spectrum.

Nicastro et al. observed the X-rays emitted by a special type of astronomical object known as a BL Lacertae (BL Lac) object. These are typically extremely bright, and have no (or very few) intrinsic spectral features - which makes it easy to detect any absorption of their emissions by other objects between them and Earth, such as filaments in the cosmic web.

The BL Lac object studied by the authors is called 1ES $1553+113$, and is more than 2,200 megaparsecs away. Nicastro and colleagues observed this target with the European Space Agency's XMM-Newton X-ray Space Telescope over several periods, for a total observation time of about 1.75 million seconds (about 20 days). They thus obtained a spectrum with an extremely high signal-to-noise ratio, which allowed them to perform highresolution spectroscopy of very weak spectral features (Fig. 1).

The authors discovered two highly statistically significant systems of absorption lines produced by helium-like oxygen (oxygen ions that have only two bound electrons) at redshifts of 0.43 and 0.36 . Redshift measures the change in wavelength that occurs when light travels over astronomical distances, and is approximately proportional to the distance of the light-emitting object from Earth. The researchers also performed an optical survey of galaxies along the sight line towards

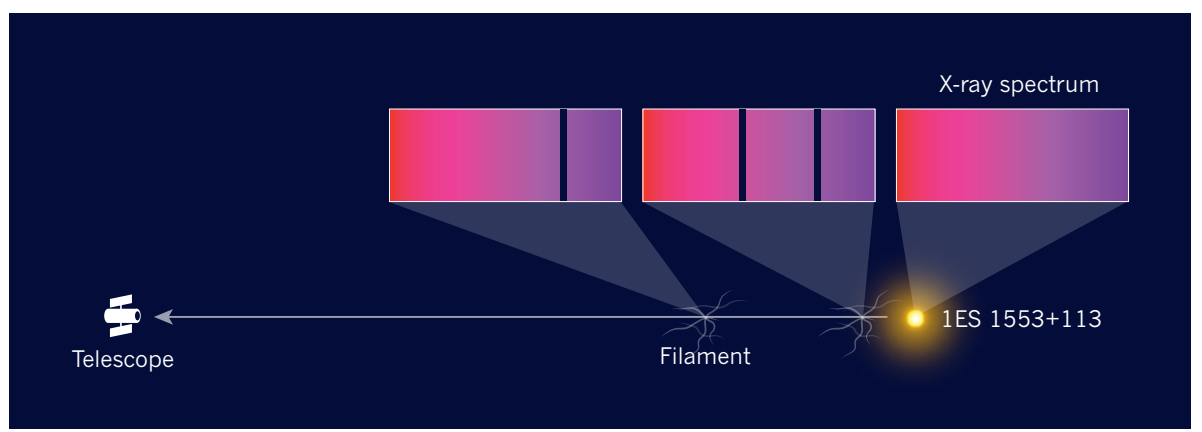

Figure 1 | The search for baryonic matter. Nicastro et al. ${ }^{6}$ used the XMM-Newton X-ray Space Telescope to detect the emission spectrum of a bright astronomical object called 1ES 1553+113. They observed lines superimposed on the spectrum, which they attribute to X-ray absorption by helium-like oxygen (oxygen ions that have just two bound electrons, not shown) in two filaments of the cosmic web located between the telescope and the emitter. The cosmic web is a massive structure composed of ordinary (baryonic) matter, such as that found in all atoms. If the authors' attribution is correct, then the finding reveals the location of a major reservoir of baryonic matter. Distances and sizes of objects not shown to scale. 
1ES 1553+113, and observed a high density of galaxies at the two redshifts associated with the absorption signals. Such densities are characteristic of the filamentary structures of the cosmic web. By combining the X-ray data with measurements of the ultraviolet emissions from 1ES 1553+113, Nicastro et al. estimated the density of the baryons associated with the X-ray absorbing features, and found that they account for $9-40 \%$ of the cosmic baryon density - suggesting that these features are a substantial reservoir of the missing baryons.

Weak X-ray absorption lines produced by baryons have been reported a few times before $^{7-8}$, but most of the results were marginal, and in some cases debatable. What is remarkable about the current work is that it represents the first time both of the expected absorption lines for helium-like oxygen have been detected together (for the absorption system at redshift 0.43 , although the statistical significance of one of the lines is marginal). The observation of two absorption lines from the same ion species is typically a good indication that the target ion species has been detected.

One concern is whether the observed X-rayabsorbing systems are truly located between Earth and 1ES 1553+113. The exact redshift of the BL Lac object is unknown; the best available estimate ${ }^{6}$ suggests that it is at least 0.41 . This value is less than the redshift of one of the X-ray absorbers, which implies that this absorber is either part of the BL Lac object, or a misidentification of something else. Nicastro et al. argue that both scenarios are unlikely, but an accurate measurement of the redshift of $1 \mathrm{ES} 1553+113$ is needed to resolve this issue.

It is also possible that the X-ray-absorbing systems are in galaxies, rather than in filamentary structures of the cosmic web - similar absorption systems have previously been detected in the Milky Way ${ }^{4}$. Nicastro and coworkers argue that this explanation is unlikely, partly because they did not find large galaxies similar to the Milky Way at the redshifts associated with the absorptions, but also because they did not detect any additional absorption lines from cold ions, which are typically found in galactic disks. These arguments are reasonable, but better observations are needed to rule out this scenario.

This type of observation, requiring more than a million seconds of exposure time, truly pushes the limits of the available instruments. Proposed space missions such as the Hot Universe Baryon Surveyor (go.nature. com/2luj4fa) and the Advanced Telescope for High-Energy Astrophysics (http://sci.esa.int/ athena/) will have much more sensitive X-ray spectrometers, and might eventually provide a complete map of the missing baryons in the cosmic web.

An alternative approach for detecting the missing baryons is to use a phenomenon known as the Sunyaev-Zel'dovich effect, in which high-energy electrons scatter off photons in the cosmic microwave background (CMB; electromagnetic radiation left over as a remnant of the Big Bang), thereby slightly distorting the CMB spectrum. High-energy electrons outside galaxies, and probably also in the filaments of the cosmic web, could produce such a distortion ${ }^{9}$, yielding a signal that indicates the presence of baryons. In the meantime, Nicastro and colleagues' findings offer a tantalizing glimpse of where the elusive missing baryons have been hiding.

Taotao Fang is in the Department of Astronomy and the Jiujiang Research Institute,
Xiamen University, Xiamen, Fujian 361005, China.

e-mail:fangt@xmu.edu.cn

1. Planck Collaboration. Astron. Astrophys. 594, 13 (2016).

2. Fukugita, M., Hogan, C. J. \& Peebles, P. J. E. Astrophys. J. 503, 518 (1998).

3. Cen, R. \& Ostriker, J. P. Astrophys. J. 514, 1 (1999)

4. Bregman, J. N. Annu. Rev. Astron. Astrophys. 45, 221-259 (2007)

5. Shull, J. M., Smith, B. D. \& Danforth, C. W. Astrophys. J. 759, 23 (2012).

6. Nicastro, F. et al. Nature 558, 406-409 (2018).

7. Fang, T., Marshall, H. L., Lee, J. C., Davis, D. S. \& Canizares, C. R. Astrophys. J. 572, L127 (2002).

8. Nicastro, F. et al. Nature 433, 495-498 (2005).

9. Hernández-Monteagudo, C. et al. Phys. Rev. Lett. 115, 191301 (2015).

\section{CLINICAL ONCOLOGY}

\section{Windows open for cancer immunotherapy}

Activating immune cells to destroy tumours is an effective strategy for treating an advanced lung cancer - but only for some people. Evidence that this approach has potential in early disease and as a combination therapy has now emerged.

\section{LIZZA E. HENDRIKS \& BENJAMIN BESSE}

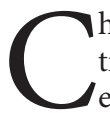
hemotherapy became the standard treatment for lung cancer in the twentieth century ${ }^{1}$. But in the past 15 years, there has been a drive to improve outcomes for people with this still-deadly disease, either through therapies that target enzymes encoded by genes harbouring cancer-driving mutations, or through immunotherapies, which activate the body's immune system to target tumours. Writing in The New England Journal of Medicine, two groups ${ }^{2,3}$ provide evidence that supports the use of immunotherapies to treat non-small-cell lung cancer (NSCLC) at different stages of the disease.

Tumour cells evade destruction by activating signals known as immune checkpoints, which deactivate immune cells called T cells ${ }^{4}$. Two immune checkpoints are the proteins cytotoxic T lymphocyte antigen 4 (CTLA-4) and programmed cell death 1 (PD-1), which are expressed by T cells themselves. Another, programmed cell death ligand 1 (PD-L1), is produced by tumour cells (Fig. 1).

Antibodies that interact with these proteins to prevent their normal activity, and so reawaken the immune system, are now used to treat metastatic NSCLC - the stage at which the cancer has spread. Antibodies that bind PD-1 or PD-L1 are sometimes successful in patients who have had treatments such as chemotherapy, but whose cancer has nonetheless progressed ${ }^{5}$. Alternatively, the anti-PD-1 antibody pembrolizumab can be used as a first-line treatment for metastatic
NSCLC when the percentage of tumour cells that express PD-L1 is high - these patients respond better to immunotherapy than to chemotherapy ${ }^{6}$.

If such immune-checkpoint-targeted antibodies (ICTs) can improve outcomes for metastatic NSCLC, could they also help to tackle early-stage disease? In the first of the current papers, Forde et al. ${ }^{2}$ carried out a pilot study to investigate whether the anti-PD-1 ICT nivolumab could be used to shrink tumours before surgery, which is a standard treatment for most cases of early-stage NSCLC.

The authors treated 21 patients with 2 doses of nivolumab 2 weeks apart, starting 4 weeks before the planned surgery date. They showed that surgery did not need to be delayed (for example, because of an adverse event with nivolumab) for any patient. The researchers anticipated that four weeks would not be enough time for the reactivated immune system to significantly shrink the tumour. Indeed, imaging revealed significant shrinkage in tumours in only two patients before surgery. However, examination of the surgically removed tumours revealed that $45 \%$ had undergone a major response to the ICT - less than $10 \%$ of the tumour cells remained alive. ICTs, unlike chemotherapy, cause inflammation and scar-tissue formation in tumours, and can therefore sometimes cause tumour growth. However, the researchers found that even two tumours that showed such growth had undergone a strong pathological response.

This level of efficacy is impressive, but needs to be further investigated by 